\title{
CIRCUSTELLAR MATTER ACTIVITY IN AE/BE HERBIG STAR MWC 614
}

\author{
N.Z. Ismailov, A.N.Adigezalzade, U.Z.Bashirova \\ N. Tusi Shamakhy Astrophysical Observatory of Azerbaijan National \\ Academy of Sciences, Y.Mamedaliyev settl., Shamakhy, Azerbaijan, AZ 5626 \\ hadigozalzade@gmail.com
}

\begin{abstract}
The results of spectral observations of the Herbig Ae/Be type star HD 179218 are presented. Two wave-like cycles of variability in the parameters of hydrogen lines $\mathrm{H} \alpha$ and $\mathrm{H} \beta$ with a characteristic time of $\sim 40$ days are revealed. The first wave of variations is deeper; the branches of decreasing and increasing the spectral parameters of the lines are more clearly expressed. At the time of the first minimum, in the profile of the emission line $\mathrm{H} \alpha$ the appearance and disappearance of additional blue and red emission components are observed. At the same time, narrow absorption components were discovered in the $\mathrm{H} \beta$ line. Possible mechanisms of the observed variability of the star are discussed.
\end{abstract}

Key words: stars: variables: Herbig $\mathrm{Ae} / \mathrm{Be}$ - stars: circumstellar matter - stars: individual - HD179218

\section{Introduction}

The Herbig Ae/Be type stars (HAe/Be) are pre-main sequence (PMS) objects of intermediate mass 2-10 $\mathrm{M}_{\odot}$ and are considered to be the progenitors of Vega type stars, which are surrounded with a residual protoplanetary disks. Spectral monitoring of individual objects has shown that in the spectra of these stars are observed variable emission and absorption lines (Praderie et al., 1986; Pogodin, 1994; Rodgers et al., 2002; Mora et al., 2004). The same features are also characteristic of classical $\mathrm{T}$ Tauri stars (CTTS) (Johns at al., 1995; Schisano et al., 2009; and references therein). It is known that in young stars, emission lines, as well as some absorption lines, are formed in the circumstellar disks or in the envelopes of the stars. Such circumstellar matter can often participate in accretion, polar outflows, winds and other forms of disk interaction with the central star. Tracking the variation in the observed spectral lines makes it possible to perform diagnostics of the physical processes that are occurring in the stellar atmosphere and in the circumstellar environment. In young stars, in particularly, these processes can directly affect the formation of the planets and their evolution. Consequently, one of the important problems in the study of the early stage of evolution of stars is the study of the characteristics of the circumstellar structure and the processes of interaction of the central star with the surrounding matter.

HD 179218 (MWC 614, Sp B9-A2) is an isolated HAe/Be type star. Despite the fact that the star is relatively bright comparatively to other HAe stars, it has been studied less. Only when the star was included in the catalog of The et al. (1994), it became the subject of active research. The circumstellar surroundings of the star were studied by IR photometry and speckle interferometry by (Millan-Gabet et al., 2001), (Prizkal et al., 1997), which did not reveal closely spaced components. Spectral studies of the star were performed by Miroshnichenko et al. (1998) and in more detail (Kozlova, 2004; Kozlova et al., 2017).

According to the classification of (Meeus et al., 2001), the spectral energy distribution (SED) of the star belongs to group I, i.e. starting with the infrared band $\mathrm{K}$ and further there is an excess of radiation excited in the dust. On the (Mendigutia et al., 2012) the profile of the line $\mathrm{H} \alpha$ is consisting of a stable single-peak structure. Perhaps the star has a close companion, about 2.5 arcsec apart (Wheelwright et al., 2010; Fedele et al., 2008) showed that the star has two dust rings at distances of $1 \mathrm{AU}$ and 20 $\mathrm{AU}$, and the space between from 1 to $6 \mathrm{AU}$ from the star filled with gas. The magnetic field of the star was measured by Hebrig et al. (2009) where on the data 2008 they have got about $51 \pm 30 \mathrm{G}$.

The purpose of this paper is to carry out monitoring of the spectral variability of the star on spectral lines of hydrogen obtained in the visual range of spectrum.

\section{Observations}

Spectral observations of the star were performed at the Cassegrain focus of the $2 \mathrm{~m}$ Karl Zayss telescope of ShAO of Azerbaijan NAS by using an echelle spectrometer constructed on the base of the spectrograph UAGS (Ismailov et al., 2013). As a light detector we have used a CCD with 530x580 elements. Observations were performed in the range $\lambda 4700-6700 \AA$. The spectral resolution is $\mathrm{R}=14000$. The mean signal to noise level in the region of the line $\mathrm{H} \alpha$ is $\mathrm{S} / \mathrm{N}=80-100$, and in the region of the line $H \beta$, is $S / N=30-40$. Reduction and calibration of the spectrograms is performed in the $\mathrm{DECH}$ programs (Galazutdinov, 1992). The method of 
observations and material processing is described in more detail in the work of Ismailov et al. (2013).

Observations were conducted for the season MaySeptember 2015. In total, 28 pairs of spectrograms were obtained for 28 nights of observations. For to control of instrument stability and position measurements the spectra of standard stars HR 7300 and HR 7734 for each night were obtained. The equivalent widths $\mathrm{EW}$, the bisector radial velocities $\mathrm{V}_{\text {bis }}$, the radial velocities at the peak of the line $\mathrm{Vp}$, the half-widths FWHM (full width at half maximum), the central depths $\mathrm{R}_{\lambda}$ (intensities) of lines $\mathrm{H} \alpha$, $\mathrm{H} \beta, \mathrm{HeI} \lambda 5876 \AA$, D1, D2 NaI, SiII $\lambda 6347,6371 \AA$, [OI] $\lambda$ $6300,6363 \AA$ and diffuse interstellar bands DIB $\lambda 5780$ and $5797 \AA$ were measured.

The average error in the intensity measurements as a function of the $\mathrm{S} / \mathrm{N}$ level was $0.4-0.5 \%$ in the region of the $\mathrm{H} \alpha$ line and up to $1 \%$ in the region of the $\mathrm{H} \beta$ line. Depending on the $\mathrm{S} / \mathrm{N}$ level, errors in equivalent widths measurements, for example, were from $3 \%$ for $\mathrm{H} \alpha$, up to $30 \%$ for [OI] $\lambda$ $6300,6363 \AA$ lines. The average error in measuring the radial velocities for individual spectral lines in the spectra of standard stars does not exceed $\pm 1.5-2.0 \mathrm{~km} / \mathrm{s}$.

\section{Results}

\subsection{The line $H \alpha$.}

This line has a complex structure which consists of an emission component that is superimposed on the core of broad photospheric absorption. The structure of the emission component varies from night to night, sometimes in both the red and blue wings of the line, additional emission components appear and disappear. Figure 1 shows all overlaying profiles of the $\mathrm{H} \alpha$ line, as well as the variation in the root-mean-square deviation $\sigma$ from the average in intensity for a given value of the radial velocity. Here (and further for other line profiles) the ordinate is given by the ratio relative intensity I/Ic, where I - the intensity at a given wavelength of the spectrum, and Ic - the continuum intensity at the same wavelength. Hence it can be seen that the main variability in the central emission occurs at the peak, and also to a lesser extent on both wings. It is also seen that the intensity varied on the blue wing is noticeably larger than on the red wing.

For radial velocities, we measured the displacement of the central peak of the emission component ( $\mathrm{Vp}$ ), the shift of the line center at half of the residual intensity (bisector velocity $\mathrm{V}_{\text {bis }}$ ). The photospheric wings of the line are very wide and at the continuum level they have a maximum displacement of $-1000 \mathrm{~km} / \mathrm{s}$ and $+1000 \mathrm{~km} / \mathrm{s}$ in blue and red wings, respectively.

Since, as in the blue and red wings of the line, additional emission components appear and disappear from time to time, which could distort the wings of the emission profile, the measured $\mathrm{V}_{\text {bis }}$ velocity could not always reflect the real displacement of the center of the common emission structure in the line $\mathrm{H} \alpha$. We also considered for the analysis the character of variation in the radial velocities at the peak of the main emission component.
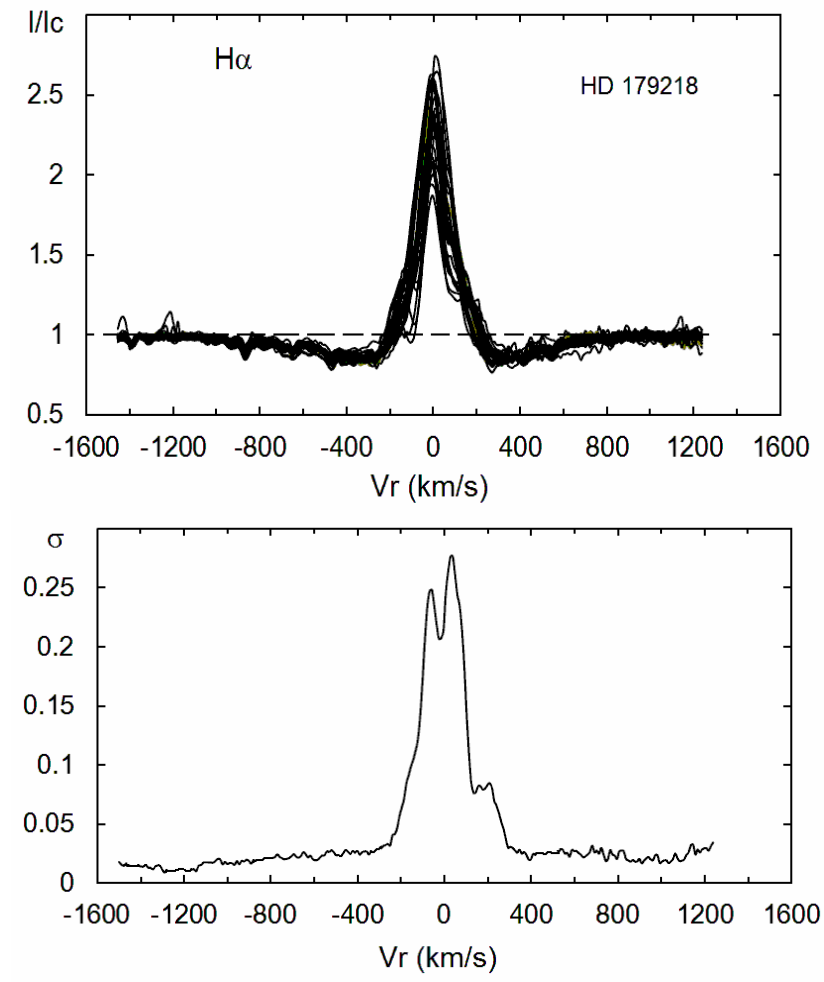

Figure 1: The overlaying profiles of the line $\mathrm{H} \alpha$ (top panel) and variation in the root-mean-square deviation $\sigma$ of the intensity versus of the radial velocities (bottom panel). Here I - is the intensity at the given wavelength of the spectrum, and Ic - the intensity of the continuum at the same wavelength.

Figure 2 shows the variation with time of the radial velocities $\mathrm{V}_{\text {bis }}$ and $\mathrm{Vp}$, as well as the equivalent widths of the emission component of the line H $\alpha$. As can be seen from these diagrams, both radial velocities and equivalent widths clearly show a wavy-like variation. The variation in the EW of the emission component demonstrates two waves of variation and return to the previous value, where first wave of event is deeper. Both of these waves of variation have duration of about 40 days, in which the general course of the reduction and then the return to the initial position is traced. Analogous variations show the radial velocities Vbis and Vp. In addition, within each 40day wave-like variations in Vbis and $\mathrm{Vp}$, variability with a small amplitude with a characteristic time of 10 to 20 days is distinguished. The total interval of variations in the parameters Vbis and Vp from peak to peak is $40 \mathrm{~km} / \mathrm{s}$ and $20 \mathrm{~km} / \mathrm{s}$, respectively, while the amplitude of a single small wave in Vbis is about $15-20 \mathrm{~km} / \mathrm{s}$, while in $\mathrm{Vp}$ it is about $10 \mathrm{~km} / \mathrm{s}$. The minima of the $\mathrm{EW}$ parameter in time coincide well with the minima of the radial velocity of the $\mathrm{Vp}$, and do not completely coincide with the minima of Vbis. It is seen that equivalent widths are not sensitive to shorter cycles of 10-20 days, which are observed at the radial velocities of the line $\mathrm{H} \alpha$. 

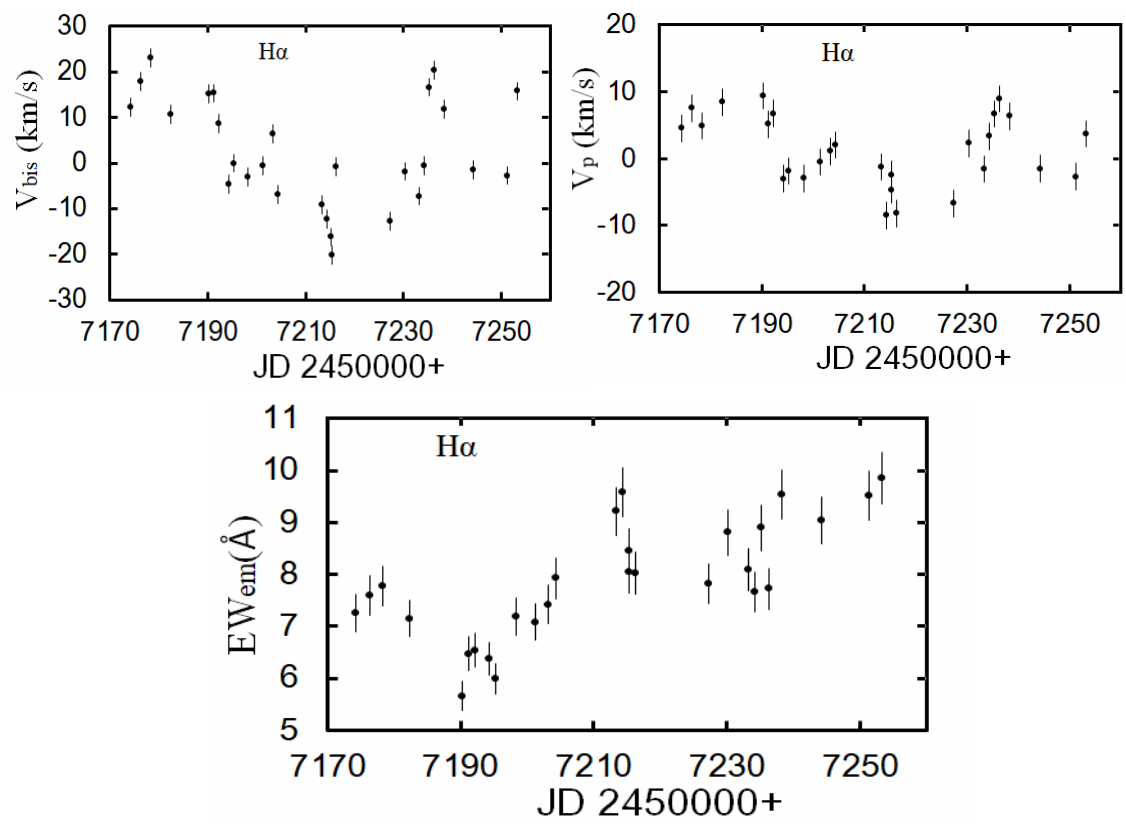

Figure 2: Variation of radial velocities Vbis (upper left panel), Vp (upper right panel) and equivalent widths (bottom panel) of emission component in the line $\mathrm{H} \alpha$. The values of the vertical bars are corresponding to the average error of parameters.
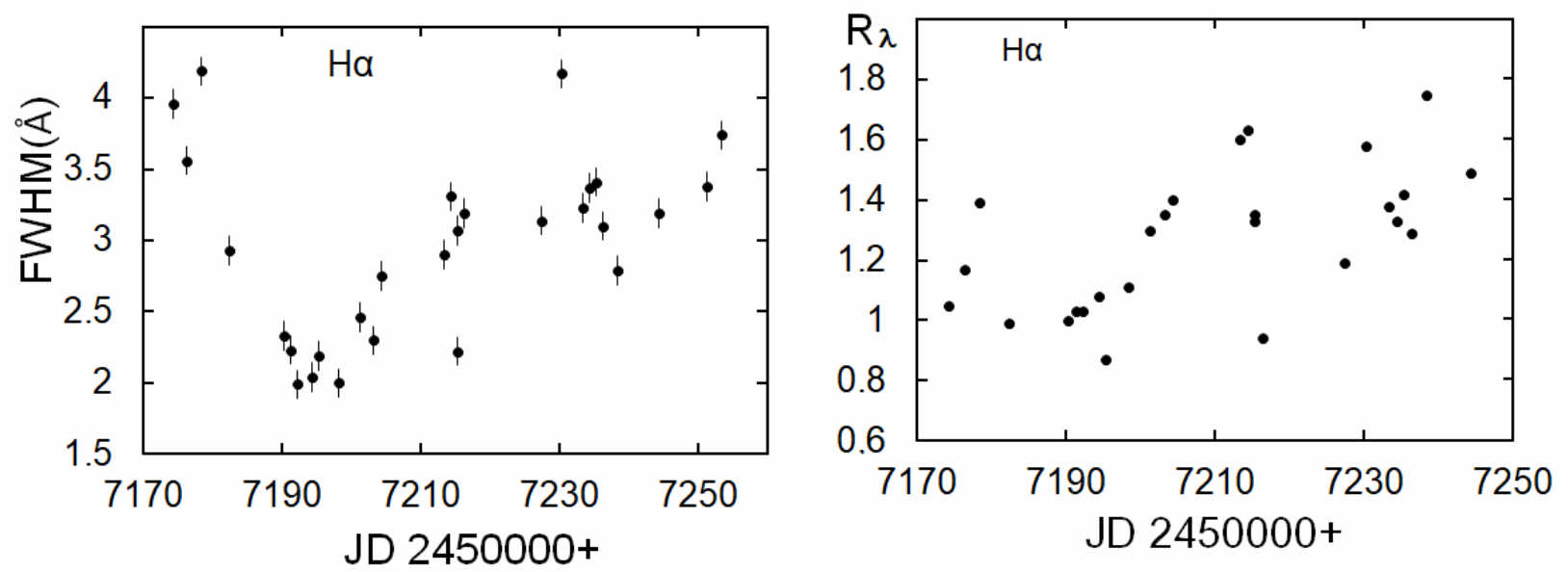

Figure 3: Variation in time of the parameters FWHM (left panel) and $\mathrm{R}_{\lambda}$ (right panel) of the emission component of the line $\mathrm{H} \alpha$.

Figure 3 shows the diagram of variations of the halfwidth FWHM (full width at half maximum) and the absolute value of the intensity $R_{\lambda}=|1-\mathrm{I} / \mathrm{Ic}|$ of the emission component of the line $\mathrm{H} \alpha$. As indicated below, the profile of the line sometimes corresponds to Type III according to (Reipurth et al.,1996). This means that the intensity of the emerging additional emission components do not exceed half of intensity of main the emission component. Therefore, the effect of these additional components on the value of the half-width should be negligible. Consequently, the variation in the parameter FWHM and $R_{\lambda}$, rather, is not directly related to the appearance of additional emission components in the line.
From Fig. 3 can be seen that a wavy-like variation in parameters FWHM and $R_{\lambda}$ is also observed. The first, deeper wave is continued about 40 days, then followed not so good distinct second wave, which also is occured about 40 days. Each of these waves is expressed by a minimum, areas of decrease and increase in the parameters (EW, FWHM, $\mathrm{R}_{\lambda}, \mathrm{Vp}$ ) of the central emission. The first wave of variation is most distinctly revealed. It is characterized by a deeper minimum, where the branch of decreasing of the line parameters was observed in the time interval JD $2457174-2457192$, and the branch of increasing - in the interval JD 2457194 -2457216 (see also Figures 2 and 3). 

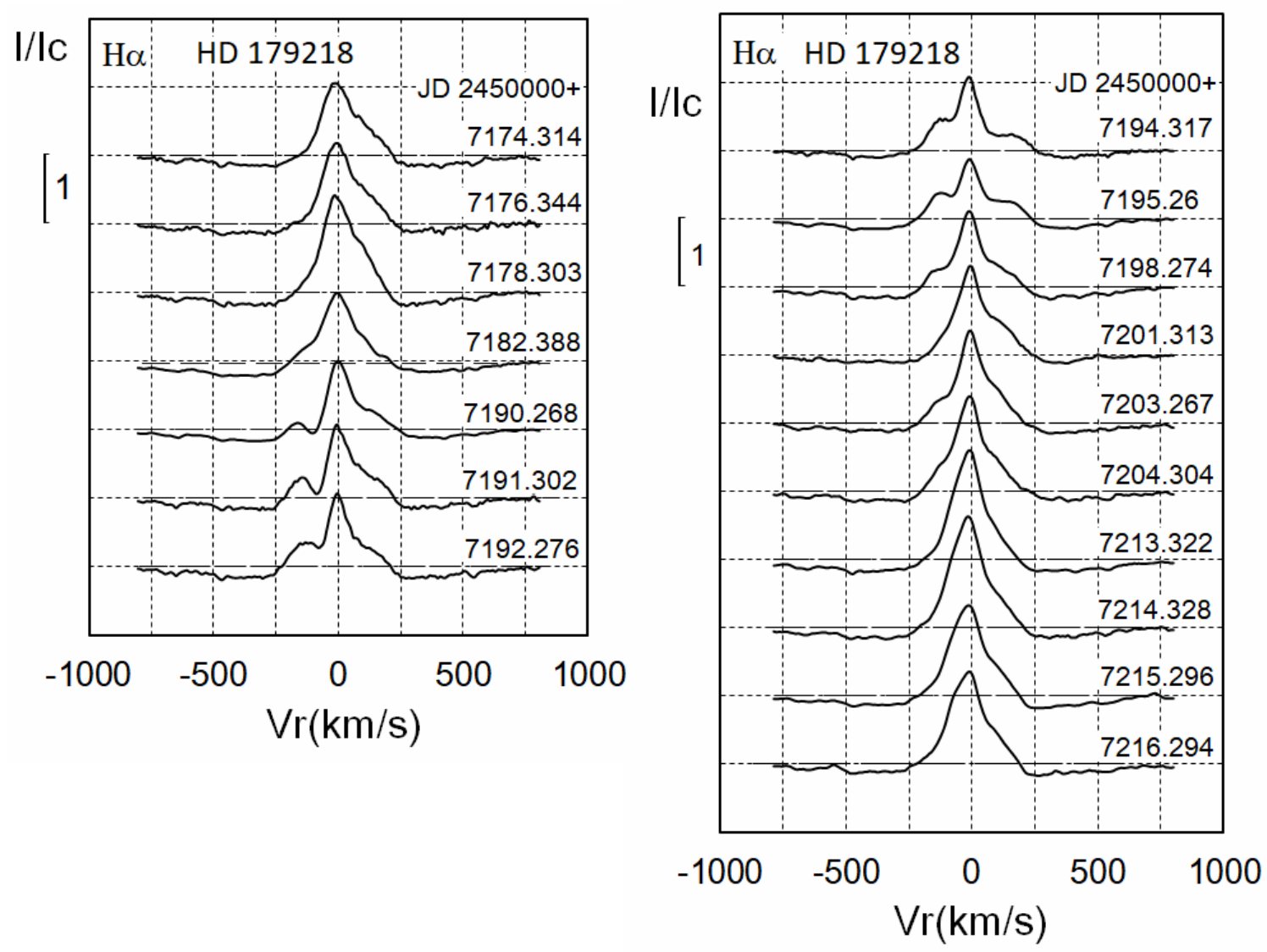

Figure 4: Variations in the profile of the emission component of the line H $\alpha$ for the descending and ascending branches over 42 days of observations in the first wave-like variations (see Fig. 4).

Figure 4 shows all profiles of the line $\mathrm{H} \alpha$, obtained in the time interval of about 42 days, when it is occured first deep minimum of variations of parameters of the line. For this time variations in the profile of the line $\mathrm{H} \alpha$ is well traced. The left panel in Fig. 4 corresponds to the time interval in which the spectral parameters are decreased, and the right panel to the time interval for increasing these parameters. As seen from the left panel of Fig. 4, starting from JD 2457190.268 over the next three nights three profiles show the appearance of a clearly marked blue and a weakerly expressed red emission components. As can be seen, in the beginning we have a profile with a central emission component. With the appearance of additional emission components on the blue and red wing, the structure of the profile is transformed from type I to type III according to the scheme of Reipurth et al. (1996). However, the obtained profile is not completely represented in type III according to the indicated classification scheme, because a central emission simultaneously produces an emission component with a blue and a red wings. The displacements of the appeared blue and red components relative to the line core are approximately symmetrical and in different dates are consisting from \pm 150 to $\pm 200 \mathrm{~km} / \mathrm{s}$.

The second panel in Fig. 4 on the right shows the results of the continuation of this series of observations, where the same split structure remains in the next 3-4 nights (JD 2457194-2457198). Such a split structure of emission component is observed until night JD 2457198 , then on the remaining nights there are profiles with single emission peak. The observed type of the line profile is unstable and was observed only at the minimum of the parameters of the main emission component (see Fig. 13). Thus, in just 8 days (JD2457190-2457198) of observations, during the first wave of decreasing in the parameters of the line $\mathrm{H} \alpha$, it was success to observe the course of the appearance and disappearance of additional emission components.

We also considered the profiles of the line $\mathrm{H} \alpha$ in the time interval of the second wave of parameter variations at JD 2457216-2457253. Among these profiles there are no profiles showing an explicit splitting into different components as in the minimum of the first wave-like of variations. There is a faint hint of the existence of an additional component on the red wing of $\mathrm{H} \alpha$ emissions only in dates JD 2457227 - 2457230. All other line profiles in this time interval are single peaks.

\subsection{Line $H \beta$}

In the line $\mathrm{H} \beta$ mainly we have observed a wide photospheric profile, on which at some nights are superimposed weak emission peaks. In most cases, such emission peaks are located symmetrically with respect to the line center, and have displacements about from -150 to $-270 \mathrm{~km} / \mathrm{s}$ in blue and from +150 to $+250 \mathrm{~km} / \mathrm{s}$ in the red wings, respectively. 

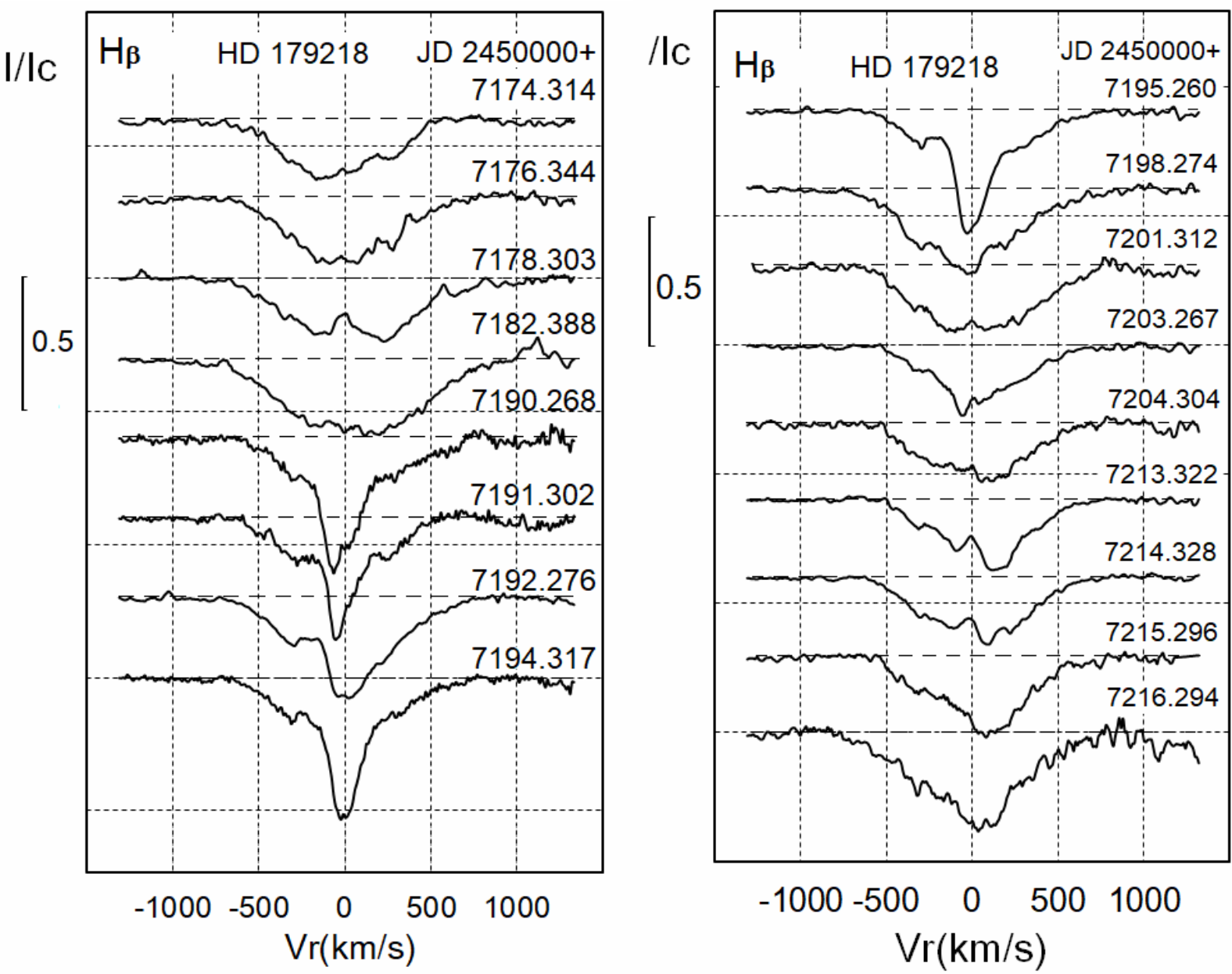

Figure 5: The profiles of the line $\mathrm{H} \beta$ obtained at the first wave of variations in the spectral parameters of the line. The left panel corresponds to the time of fall, the second panel, the increase in the spectral parameters of the line.

Among the obtained profiles of the line $H \beta$, a great interest is represented the profiles and spectral parameters, obtained during of the first wave-like decreasing. In Fig. 5 is shown profiles of the line $\mathrm{H} \beta$, which are obtained on dates, when the spectral parameters in the line $\mathrm{H} \alpha$ were degreased during for the first wave-like variation (JD 2457174 - 2457216). At the beginning, the wide photospheric profiles of the line $\mathrm{H} \beta$ with a smooth core and without any special details is observed. As can be seen, with a minimum of the spectral parameters, in JD 2457190-2457195, a significant variation in the profile of the $\mathrm{H} \beta$ line is observed - the depth of the line increases, the core becomes narrow and deep, and a weak emission component is superimposed on the wide wings. Such a structure is observed at the line $\mathrm{H} \beta$ just at the time when an additional emission component appears in the line $\mathrm{H} \alpha$ (see Fig. 4). Such a coincidence by the dates of these events in the lines $\mathrm{H} \alpha$ and $\mathrm{H} \beta$ indicates that in both cases is operated a common mechanism of variability. From the right panel of Fig. 5 shown that after leaving the minimum at JD 2457198 , the profile of the $\mathrm{H} \beta$ line gradually returns to its original position.

In the Figure 6 shown the diagrams of the time variations in the spectral parameters $\mathrm{Vp}$ - the radial velocity of the deepest absorption component, FWHM- absorption half-width, EW-equivalent width absorption, $\mathrm{R}_{\lambda_{-}}$depth (intensity) of the line $\mathrm{H} \beta$. As you can see, here too, with the first wave of variations in JD 2457174 2457216, a significant variations in the specified line parameters is noticeable. The equivalent width of absorption is increased, the FWHM is sharply reduced, the intensity of the line has increased. The radial velocities of the line peak over the whole observation season give a significant shift from -100 to $+100 \mathrm{~km} / \mathrm{s}$. The wave-like variation is best observed here in the parameter $R_{\lambda}$.

Figure 6 shows the diagrams of the variations in the spectral parameters $\mathrm{Vp}$-the radial velocity of the deepest absorption component, FWHM-absorption half-width, EW-equivalent absorption width, $\mathrm{R} \lambda$-depth (intensity) of the $\mathrm{H} \beta$ line with time. As you can see, here too, with the first wave of variations in JD 2457174 - 2457216, a significant variation in the specified line parameters is noticeable. The equivalent width of absorption is increased, the half-width is sharply reduced, the intensity of the line has increased. The radial velocities of the line peak over the whole observation season give a significant shift from -100 to $+100 \mathrm{~km} / \mathrm{s}$. The wave-like variation is best observed here in the parameter $\mathrm{R}_{\lambda}$ of the line. 

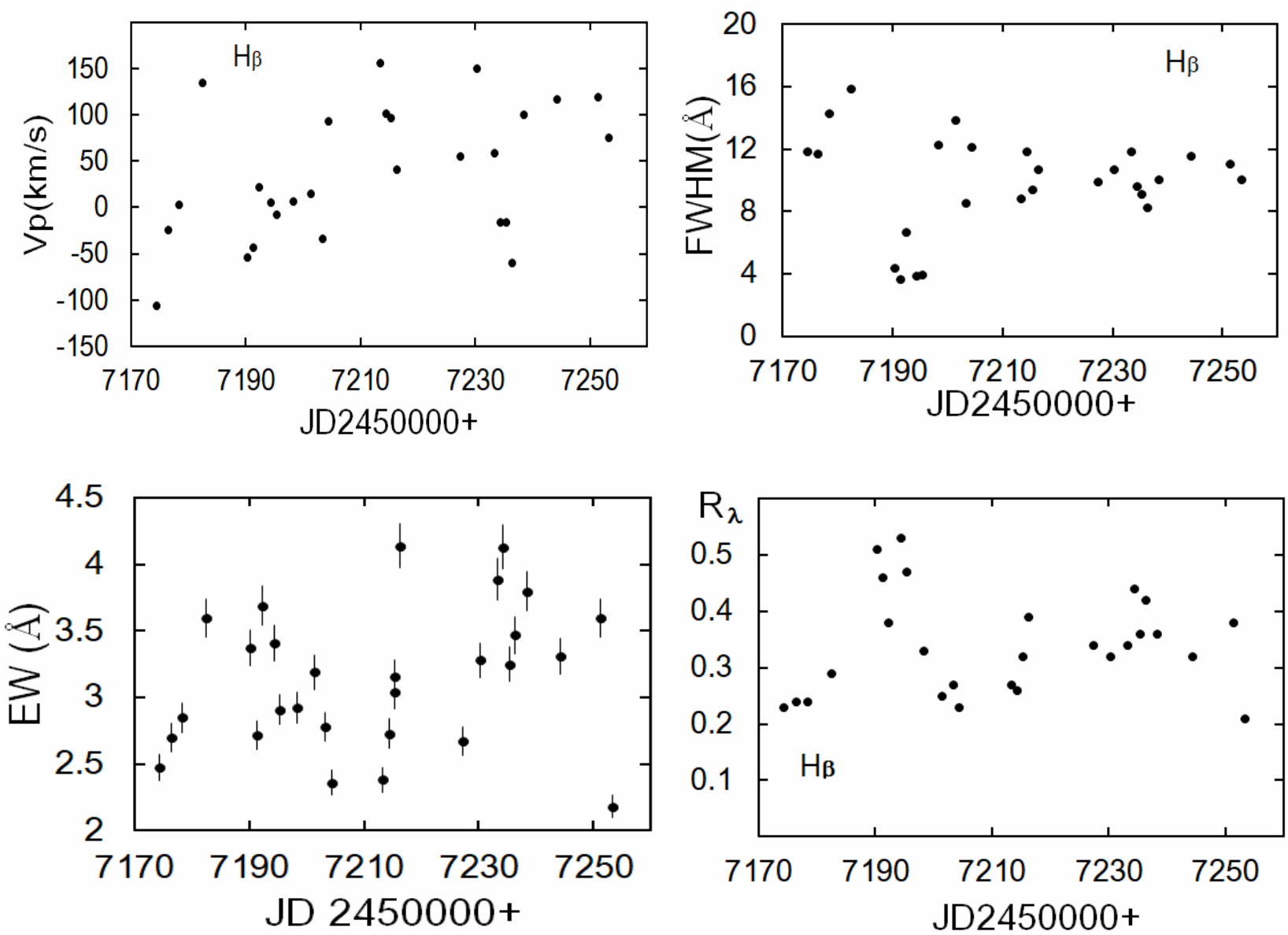

Figure 6: The time variation of the radial velocity of the peak Vp (upper left panel), half width FWHM (upper right panel), equivalent width EW (lower left panel) and intensity $\mathrm{R}_{\lambda}$ (lower right panel) of the line $\mathrm{H} \beta$.

\section{Conclusion}

The rotation velocity of HD 179218 in (Bernacca et al., 1970) is $60 \mathrm{~km} / \mathrm{s}$, and (Guimaraes et al., 2006) gives the value $v \sin \mathrm{i}=72 \pm 5 \mathrm{~km} / \mathrm{s}$. According to (Dent et al., 2005) the angle of inclination to the axis of rotation of the star is about $40^{\circ}$. Then, if we consider the observable minimum characteristic time equal to 10 days, for the rotation velocity of the star we obtain $v=112 \pm 8 \mathrm{~km} / \mathrm{s}$ and for the star radius $\mathrm{R}^{*}$ - about $22 \mathrm{R}_{\odot}$, which is not reasonable and differs significantly from the data of (Alecian et al., 2008) ( 4.8 $\left.\mathrm{R}_{\odot}\right)$. An even greater discrepancy is obtained for the radius if we take the angle $\mathrm{i}=20^{\circ}$, as suggested by Leinert et al. (2004). This means that the observed cycle of about 10 days can not be a period of axial rotation of the star. Remaind that the characteristic time of 10 days is obtained from the variation in the radial velocities of the peak of dominant emission component and the intensity of the line. Therefore, it should be assumed that it arises in the outer parts of the disk. However, if the observed 10-day activity is related to the axial rotation of the disk, it can be assumed that such a variation could occur at the boundary between the matter accretion and outflow. Then one of the assumptions of the cause of the observed variations in the emission lines of the star may be the existence of a stellar magnetosphere. In favor of the possibility of the existence of magnetospheric accretion, the star is also proposed in the work of (Mendigutia et al., 2017). The main indicator existence of the magnetosphere of the star is the strength of magnetic field. In classical $T$ Tauri stars, for which the presence of the magnetosphere is assumed, the magnitude of the magnetic field is several KGs (see, for example, Bouvier et al., 2007). However, the result of measuring the magnetic field of star HD 179218 shows the existence of a weak magnetic field (Hebrig et al., 2009).

It is also possible that a star can be a spectral-binary or multiple system. In fact, it is difficult to explain the observed wave-like variation of the radial velocities and

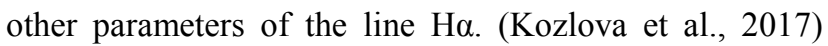
showed that the dependence of the brightness $\mathrm{V}$ on the color index V-I has two separate distributions. This fact is accepted by the authors in favor of the binarity of the star. The time of our observations from May to September 2015 corresponds to the minimum of the 4000-day cycle of variability founded by (Kozlova et al., 2017). Therefore, the observed features of the variation in the spectrum in the line $\mathrm{H} \alpha$ can be related to the moment of the star's stay at the minimum of the 4000-day cycle. Then the results obtained by us, perhaps, are a kind of unique event and can be observed only in the minima of the 4000day cycle. Our observations have shown that in order to 
elucidate these questions it is necessary to perform a more dense series of photometric and spectral observations of the star.

Based on the results obtained in this paper, we can draw the following conclusions:

1. There are two wave-like variations lasting about 40 days each, in the parameters of the emission component of the $\mathrm{H} \alpha$ line in the spectrum of the star HD 179218. The first wave is deeper, the second is relatively less significant, and possibly distorted by fluctuations.

2. An unusual variation in the profile of the $\mathrm{H \alpha}$ line near the minimum in the descending and ascending branches of the first wave of variations in the spectral parameters was observed. At the entrance to the minimum and during the passage of the minimum, appearance and disappearance of additional emission components in the line $\mathrm{H} \alpha$ was detected. The displacement of each components corresponds to approximately $\pm 150 \mathrm{~km} / \mathrm{s}$. During the rest of the observation time, such profiles were no longer detected.

3 . With the first wave of variations, the parameters of the $H \beta$ line showed a synchronous variation in time with the line $\mathrm{H} \alpha$. At the time of appearance of additional emission components near the line $\mathrm{H} \alpha$, narrower and deeper components appear at the line $\mathrm{H} \beta$.

4. Within each 40-days wave-like variations, a small amplitude fluctuation of the radial velocities of the peak of the emission component and the intensity of the $\mathrm{H} \alpha$ line with a characteristic time of about 10-20 days is observed. Also, the half-widths and intensities of the line $\mathrm{H} \beta$ more clearly demonstrate the existence of 10-20 day wave-like variations.

\section{References}

1. Alecian E., Catala C., Wade G. A. et al.: 2008, MNRAS, 385, 391.

2. Bouvier J., Alencar S.H.P., Harries T.J. et al.: 2007, in: Protostar and Planets V, Univ. Arizona Press, p. 479.

3. Dent W.R.F., Greaves J.S., Coulson I.M.: 2005, MNRAS, 359, 663.
4. Fedele D., Van den Ancker M.E., Acke B. et al.: 2008, Astron. and Astrophys., 491, 809.

5. Galazutdinov G.A.: 1992, Prepr. of the Special Astrophysical Observatory, №.92.

6. Guimaraes M.M., Alencar S.H.P., Corradi W.J.B., Vieira S.L.A.: 2006, Astron. and Astrophys., 457, 581.

7. Johns C.M., \& Basri G.: 1995, AJ, 109, 2800.

8. Hebrig S., Stelzer B., Schöller M. et al.: 2009, Astron. and Astrophys., 502, 283.

9. Ismailov N.Z., Bahaddinova G.R., Kalilov O.V., Mikailov Kh.M.: 2013, Astrophys.Bull., 68, №2, 196.

10. Kozlova O.V.: 2004, Astrophys., 47, № 3, 287.

11. Kozlova O.V., Alekseev I.Yu.: 2017, ASP Conf. Ser., 510, 153.

12. Leinert Ch., van Boekel R., Waters L.B.F.M. et al.: 2004, Astron. and Astrophys., 423, 537.

13. Mora A., Eiroa C., Natta A. et al.: 2004, $A \& A$, 419, 225.

14. Miroshnichenko A.S., Bjorkman K.S., Mulliss C.L. et al.: 1998, PASP, 110, 883.

15. Mendigutía I., Eiroa C., Montesinos B., Mora A., Oudmaijer R.D., Merín B., Meeus G.: 2011, $A \& A$, 529, A34.

16. Mendigutia I., Oudmaijer R.D., Mourard D., Muzerolle J.: 2017, Monthly Notices Royal Astron. Soc., 464, 1984.

17. Mendigutia I., Mora A, Montesinos B. et al.: 2012, $A \& A, \mathbf{5 4 3}, \mathrm{A} 59$.

18. Millan-Gabet R., Schloerb F.P., Traub W.A.: 2001, Ap.J., 546, 358.

19. Praderie F., Simon T., Catala C., Boesgaard A.M.: 1986, ApJ, 303, 311.

20. Pogodin M.A.: 1994, $A \& A, \mathbf{2 8 2}, 141$.

21. Pirzkal N., Spillar E.J., Dyck H.M.: 1997, Ap.J., 481, 392.

22. Rodgers B.,Wooden D.H., Grinin V. et al.: 2002, ApJ, 564, 405.

23. Schisano E., Covino E., Alcalá J.M. et al.: 2009, $A \& A, \mathbf{5 0 1}, 1013$.

24. The P.S., De Winter D., Perez M.R.: 1994, A\&ASS, 104, 315.

25. Wheelwright H.E., Oudmaijer R.D., Goodwin S.P.: 2010, Monthly Notices Royal Astron. Soc., 401, 1199. 